\title{
CUMULATIVE BEAM BREAKUP IN LINEAR ACCELERATORS WITH ARBITRARY BEAM CURRENT PROFILE*
}

\author{
J. R. Delayen ${ }^{\dagger}$, Thomas Jefferson National Accelerator Facility, Newport News, VA 23606, USA
}

\section{Abstract}

An analytical formalism for the solution of cumulative beam breakup in linear accelerators with arbitrary beam current profile is developed. It is applied to obtain an expression for the transverse displacement of trains of finite bunches. The same formalism is used to investigate the beam breakup-enhanced displacement of beams caused by the misalignment of the deflecting structures or focusing elements.

\section{INTRODUCTION}

Cumulative beam breakup (BBU) has been extensively investigated in the past by many authors; the present work is a continuation a previous investigation of $\mathrm{BBU}$ in the case of bunches of finite length [1]. That previous investigation was limited to steady state analysis, and we extend it here to the analysis of the transient behavior of arbitrary beams and, in particular, of finite trains of bunches of finite length. This extension is motivated by the increasing interest in pulsed high-current superconducting accelerators, and could also be applied to the analysis of long-range effects in linear colliders.

\section{EQUATION AND GENERAL SOLUTION}

In a continuum approximation, the transverse motion of a beam under the influence of focusing and BBU can be modeled by [1]

$\left[\frac{1}{\beta \gamma} \frac{\partial}{\partial \sigma}\left(\beta \gamma \frac{\partial}{\partial \sigma}\right)+\kappa^{2}\right] x(\sigma, \zeta)=\varepsilon \int_{-\infty}^{\zeta} d \zeta_{1} w\left(\zeta-\zeta_{1}\right) F\left(\zeta_{1}\right) x\left(\sigma, \zeta_{1}\right)$ where $\beta$ and $\gamma$ are the usual velocity and energy parameters; $\sigma=s / \mathbf{L}$, is the distance from the front of the accelerator normalized to the accelerator length; $\kappa$ is the normalized focusing wave number; $\left.\zeta=\omega\left(t-\int d s / \beta c\right)\right)$, is the time made dimensionless by the frequency $\omega$ and measured after the arrival of the head of the beam at location $\sigma ; F(\zeta)=I(\zeta) / \bar{I}$, the current form factor, is the instantaneous current divided by the average current; $w(\zeta)$ is the wake function, which, in the case of a single dipole mode, is assumed to be $w(\zeta)=U(\zeta) \sin \zeta e^{-\zeta / 2 Q} ; \varepsilon$ is the coupling strength between the beam and the dipole mode, and includes properties of the beam and the deflecting mode of the accelerating structure.

Without loss of generality we will assume a coasting beam, and constant BBU and focusing strengths along the accelerator. Under some reasonable assumptions, these restrictions can often be relaxed and suitable coordinate

\footnotetext{
* This work was supported by the U.S. DOE Contract No DE-AC0584-ER40150.

†delayen@jlab.org
}

transformations can be introduced to transform the full equation of motion to that of a coasting beam with constant parameters [2].

Under these assumptions, the equation of motion becomes

$\left(\frac{\partial^{2}}{\partial \sigma^{2}}+\kappa^{2}\right) x(\sigma, \zeta)=\varepsilon \int_{-\infty}^{\zeta} d \zeta_{1} w\left(\zeta-\zeta_{1}\right) F\left(\zeta_{1}\right) x\left(\sigma, \zeta_{1}\right)$.

Applying to Equation (1) the Laplace transform with respect to the variable $\sigma: \mathcal{L}[x(\sigma, \zeta)]=x^{\dagger}(p, \zeta)$, and assuming, for the sake of simplicity, that the beam enters the accelerator off, but parallel to, the axis, i.e.

$$
x(\sigma=0, \zeta)=x_{0}(\zeta), x^{\prime}(\sigma=0, \zeta)=0,
$$

we obtain

$$
\begin{aligned}
x^{\dagger}(p, \zeta) & =\frac{p}{p^{2}+\kappa^{2}} x_{0}(\zeta) \\
& +\frac{\varepsilon}{p^{2}+\kappa^{2}} \int_{-\infty}^{\zeta} d \zeta w\left(\zeta-\zeta_{1}\right) F(\zeta) x^{\dagger}\left(p, \zeta_{1}\right)
\end{aligned}
$$

Using the right-hand side of this equation to replace $x^{\dagger}(p, \zeta)$ in the integral yields

$$
\begin{aligned}
x^{\dagger}(p, \zeta)= & \frac{p}{p^{2}+\kappa^{2}} x_{0}(\zeta) \\
& +\frac{p \varepsilon}{\left(p^{2}+\kappa^{2}\right)^{2}} \int_{-\infty}^{\zeta} d \zeta_{1} w\left(\zeta-\zeta_{1}\right) F\left(\zeta_{1}\right) x_{0}\left(\zeta_{1}\right) \\
& +\frac{p \varepsilon^{2}}{\left(p^{2}+\kappa^{2}\right)^{2}} \int_{-\infty}^{\zeta} d \zeta_{1} w\left(\zeta-\zeta_{1}\right) F\left(\zeta_{1}\right) \\
& \times \int_{-\infty}^{\zeta_{1}} d \zeta_{2} w\left(\zeta_{1}-\zeta_{2}\right) F\left(\zeta_{2}\right) x^{\dagger}\left(p, \zeta_{2}\right)
\end{aligned}
$$

The same substitution for $x^{\dagger}(p, \zeta)$ can be applied to this last expression and repeated indefinitely. This results in the following series expansion for $x^{\dagger}(p, \zeta)$ :

$$
\begin{gathered}
x^{\dagger}(p, \zeta)=\sum_{n=0}^{\infty} \frac{p \varepsilon^{n}}{\left(p^{2}+\kappa^{2}\right)^{n+1}} h_{n}(\zeta) \\
h_{0}(\zeta)=x_{0}(\zeta), h_{n+1}(\zeta)=\int_{-\infty}^{\zeta} h_{n}\left(\zeta_{1}\right) F\left(\zeta_{1}\right) w\left(\zeta-\zeta_{1}\right) d \zeta_{1} .
\end{gathered}
$$

Applying the inverse Laplace transform to Equation (2) gives the transverse displacement $x(\sigma, \zeta)$ for arbitrary time $\zeta$, location $\sigma$, beam current profile $F(\zeta)$, wake function $w(\zeta)$, and time dependence of the beam offset at the entrance of the accelerator $x_{0}(\zeta)$ :

$$
\begin{aligned}
& x(\sigma, \zeta)=\sum_{n=0}^{\infty} \frac{1}{n !}\left(\frac{\varepsilon \sigma}{2 \kappa}\right)^{n} \sqrt{\frac{\pi \kappa \sigma}{2}} J_{n-\frac{1}{2}}(\kappa \sigma) h_{n}(\zeta) \\
& h_{0}(\zeta)=x_{0}(\zeta), h_{n+1}(\zeta)=\int_{-\infty}^{\zeta} h_{n}\left(\zeta_{1}\right) F\left(\zeta_{1}\right) w\left(\zeta-\zeta_{1}\right) d \zeta_{1}
\end{aligned}
$$


Several remarks can be made about the expression for $x(\sigma, \zeta)$. First, it is a series expansion in the powers of the ratio of BBU coupling to focusing strength; when the BBU is weak or the focusing strong, only a few terms need to be kept. Second, the dependence on the current profile and wake function is limited to the functions $h_{n}(\zeta)$. Third, the functions $h_{n}(\zeta)$ depend only on the current or bunch profiles, not on their magnitude; that magnitude is included in the coefficient $\varepsilon$.

\section{EXAMPLES}

\subsection{Single, very short bunch}

This formalism can be easily applied to the case of a single very short bunch entering the accelerator with a transverse offset $x_{0}$. By very short we assume that the bunch length is much shorter than the period of the dipole mode, so the wake function can be assumed to be linear: $w(\zeta)=\zeta$. The functions $h_{n}(\zeta)$ can be easily calculated: $h_{n}(\zeta)=x_{0} \frac{\zeta^{2 n}}{(2 n) !}$, to give, for large $\sigma$ where an asymptotic form for the Bessel functions can be used,

$$
\begin{aligned}
x(\sigma, \zeta) & =x_{0} \sum_{n=0}^{\infty} \cos \left[\kappa \sigma-\frac{n \pi}{2}\right] \frac{1}{n !(2 n) !}\left(\frac{\varepsilon \sigma \zeta^{2}}{2 \kappa}\right)^{n} \\
& =x_{0}\left[\cos (\kappa \sigma)+\frac{\varepsilon \sigma \zeta^{2}}{4 \kappa} \sin (\kappa \sigma)+\ldots\right] .
\end{aligned}
$$

This result was first obtained in [3].

\subsection{Finite train of finite bunches}

A more interesting application of Equation (3) is the investigation of the transient behavior of a finite train of finite bunches. We assume that the bunches are separated by $\zeta=\omega \tau$, have a length $\alpha \omega \tau$, and that the charge has a constant profile within each bunch, so that $F(\zeta)=U(\zeta) \sum_{k=-\infty}^{+\infty} e^{-i k \pi \alpha} \frac{\sin (k \pi \alpha)}{k \pi \alpha} e^{i \frac{2 \pi}{\omega \tau} \zeta}$. We assume that the wakefield is due to a single dipole mode: $w(\zeta)=U(\zeta) \sin \zeta e^{-\zeta / 2 Q}$; and we look at the transverse displacement of a particle located within bunch $M+1$, and a (normalized) time $\alpha \omega \tau \phi$ behind the head of that bunch, so that $\zeta=\omega \tau[M+\alpha \phi] ; \phi=0$ is the head of the bunch while $\phi=1$ is its tail.

From Equation (3), the transverse displacement, at location $\sigma$, for a particle arriving at time $\zeta=\omega \tau[M+\alpha \phi]$ is, to second order,

$x(\sigma, \zeta)=x_{0}\left[\cos (\kappa \sigma)+\frac{h_{1}(\zeta)}{x_{0}} \frac{\varepsilon \sigma}{2 \kappa} \sin (\kappa \sigma)+\ldots ..\right]$,

where

$$
\begin{aligned}
& \frac{h_{1}(\zeta)}{x_{0}}=\frac{1}{2 \alpha} \times \\
& \left\{\begin{array}{l}
\frac{1}{1+\frac{i}{2 Q}}\left\{1-e^{\omega \tau \alpha\left(\phi-\frac{1}{2}\right)\left(-\frac{1}{2 Q}+i\right)} \frac{\sin \left[\frac{\omega \tau}{2}\left(1+\frac{i}{2 Q}\right)(1-\alpha)\right]}{\sin \left[\frac{\omega \tau}{2}\left(1+\frac{i}{2 Q}\right)\right]}\right\}+\mathrm{cc} \\
-\frac{e^{-\zeta\left(-\frac{1}{2 Q}+i\right)}}{1+\frac{i}{2 Q}}\left\{1-e^{\omega \tau \alpha\left(\phi-\frac{1}{2}\right)\left(-\frac{1}{2 Q}+i\right)} \frac{\sin \left[\frac{\omega \tau}{2}\left(1+\frac{i}{2 Q}\right)(1-\alpha)\right]}{\sin \left[\frac{\omega \tau}{2}\left(1+\frac{i}{2 Q}\right)\right]}\right\}+\mathrm{cc}
\end{array}\right\}
\end{aligned}
$$

An example is shown in Figure 1.

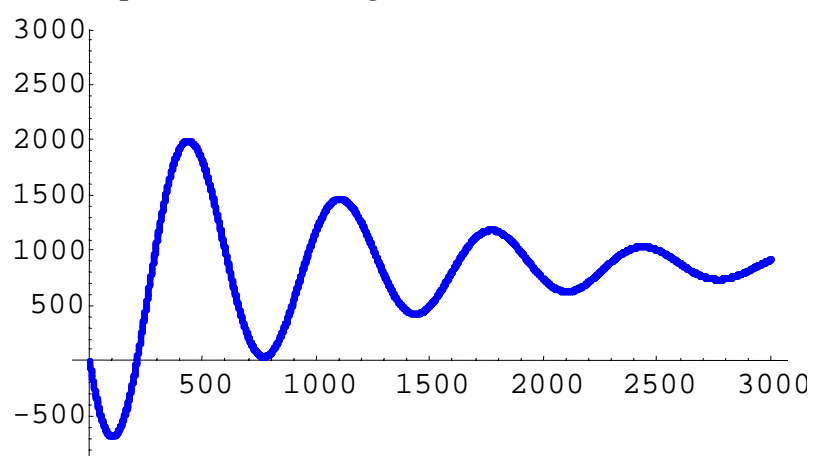

$-1000$

Figure 1: Function $h_{1}(\zeta) / x_{0}$ for the front of the bunch $(\phi=0)$ as a function of bunch number for $Q=10000, \alpha=0.1, \omega \tau=6 \pi \times 1.0005$

\section{MISALIGNED ACCELERATING STRUCTURES}

The same method can be used to analyze the beam displacement caused by a misalignment of the accelerating structures and coupling to the dipole modes. The transverse equation of motion becomes

$\frac{\partial^{2}}{\partial \sigma^{2}} x+\kappa^{2} x=\varepsilon \int_{-\infty}^{\zeta} d \zeta^{\prime} w\left(\zeta-\zeta^{\prime}\right) F\left(\zeta^{\prime}\right)\left[x\left(\sigma, \zeta^{\prime}\right)-d(\sigma)\right]$,

where $d(\sigma)$ is the displacement of the accelerating structure at location $\sigma$, or more precisely the transverse displacement of the axis of the dipole mode with respect to the beam line defined by the focusing elements. Applying the Laplace transform yields

$$
\begin{aligned}
x^{\dagger}(p, \sigma)= & -\frac{\varepsilon d^{\dagger}(p)}{p^{2}+\kappa^{2}} \int_{-\infty}^{\zeta} d \zeta^{\prime} w\left(\zeta-\zeta^{\prime}\right) F\left(\zeta^{\prime}\right) \\
& +\frac{\varepsilon}{p^{2}+k^{2}} \int_{-\infty}^{\zeta} d \zeta^{\prime} w\left(\zeta-\zeta^{\prime}\right) F\left(\zeta^{\prime}\right) x^{\dagger}\left(p, \zeta^{\prime}\right),
\end{aligned}
$$

where we have assumed $x_{0}(\zeta)=0$ and $x_{0}^{\prime}(\zeta)=0$, i.e. the beam enters the accelerator on, and parallel to, the axis. The right-hand side of the above equation can be used to replace $x^{\dagger}(p, \sigma)$ in the second integral, and the same process can be repeated indefinitely to give 


$$
x^{\dagger}(p, \zeta)=d^{\dagger}(p) \sum_{n=1}^{\infty} \frac{(-1)^{n} \varepsilon^{n}}{\left(p^{2}+\kappa^{2}\right)^{n}} \quad H_{n}(\zeta),
$$

with $H_{0}(\zeta)=1, H_{n+1}(\zeta)=\int_{-\infty}^{\zeta} d \zeta^{\prime} w\left(\zeta-\zeta^{\prime}\right) F\left(\zeta^{\prime}\right) H_{n}\left(\zeta^{\prime}\right)$.

Applying the inverse Laplace transform gives the following expression for the transverse displacement of the beam

$$
x(\sigma, \zeta)=\sum_{n=1}^{\infty}(-1)^{n} \varepsilon^{n} H_{n}(\zeta) \int_{0}^{\sigma} d u i_{n}(u) d(\sigma-u),
$$

where

$i_{n}(\sigma)=\mathcal{L}^{-1}\left[\frac{1}{\left(p^{2}+\kappa^{2}\right)^{n}}\right]=\frac{1}{(n-1) !}\left(\frac{\sigma}{2 \kappa}\right)^{n-1} \frac{1}{\kappa} \sqrt{\frac{\pi \kappa \sigma}{2}} J_{n-\frac{1}{2}}(\kappa \sigma)$.

While a complete determination of $x(\sigma, \zeta)$ could be obtained if one had complete knowledge of $d(\sigma)$, only a statistical determination is often available for the latter or needed for the former. The mean square displacement of the beam at location $\sigma$ and time $\zeta$ is given by

$$
\left\langle x^{2}(\sigma, \zeta)\right\rangle=\sum_{m=1}^{\infty} \sum_{n=1}^{\infty}(-\varepsilon)^{m+n} x_{m n}^{2}(\sigma) H_{m}(\zeta) H_{n}(\zeta),
$$

where $x_{m n}^{2}(\sigma)=\int_{0}^{\sigma} \int_{0}^{\sigma} d u d v i_{m}(\sigma-u) i_{n}(\sigma-v) R_{d}(u-v)$, and $R_{d}(u-v)$ is the autocorrelation function of $d(\sigma)$.

If one assumes that the structures have a length $\sigma_{0}$, are displaced parallel to the axis, that their displacements are uncorrelated from one structure to the others, and follow a probability density of standard deviation $d_{0}$, then the autocorrelation function $R_{d}(u-v)$ is

$$
\left.R_{d}(u-v)=d_{0}^{2} \sigma_{0}\left(1-\mid \frac{u-v}{\sigma_{0}}\right)\right) \text { for }-\sigma_{0}<u-v<\sigma_{0} .
$$

In the limits $\sigma \gg \sigma_{0}$ and $\kappa \sigma_{0} \ll 1$, a simpler model for $R_{d}(u-v)$ that allows closed-form calculation of the functions $x_{m n}^{2}(\sigma)$ is $R_{d}(u-v)=d_{0}^{2} \sigma_{0} \delta(u-v)$. For large values of $\kappa \sigma$, the asymptotic form of $i_{m}(\sigma)$ can be used: $i_{n}(\sigma) \sim \frac{1}{(n-1) !} \quad \frac{1}{2^{n-1} \kappa^{n}} \quad \sigma^{n-1} \cos \left[\kappa \sigma-\frac{n \pi}{2}\right]$.

It is easy to show that the main contributions to $\left\langle x^{2}(\sigma, \zeta)\right\rangle$ will be from $m=n$ :

$$
x_{n n}^{2}(\sigma)=\frac{2 d_{0}^{2} \sigma_{0}}{[(n-1) !]^{2}(2 n-1)(2 \kappa)^{2 n}} \sigma^{2 n-1} .
$$

Applying this formalism to the case of a single, very short bunch, the functions $H_{n}(\zeta)$ can be easily obtained:

$$
\begin{aligned}
& H_{n}(\zeta)=\frac{\zeta^{2 n}}{(2 n) !} \text {, and } \\
& \left\langle x^{2}(\sigma, \zeta)\right\rangle=\sum_{n=1}^{\infty} \varepsilon^{2 n} \frac{2 d_{0}^{2} \sigma_{0} \sigma^{2 n-1}}{[(n-1) !]^{2}(2 n-1)(2 \kappa)^{2 n}} \frac{\zeta^{4 n}}{[(2 n) !]^{2}} .
\end{aligned}
$$

Keeping only the first two terms gives

$$
\left\langle x^{2}(\sigma, \zeta)\right\rangle=\frac{d_{0}^{2} \sigma_{0} \varepsilon^{2} \sigma \zeta^{4}}{8 \kappa^{2}}\left[1+\frac{\varepsilon^{2} \sigma^{2} \zeta^{4}}{1728 \kappa^{2}}\right],
$$

a result first obtained in [3]. The front of the bunch $(\zeta=0)$ is not displaced but the bunch develops a tail that varies as $\zeta^{2}$ and whose magnitude increases as $\kappa^{-1} \varepsilon \sigma^{1 / 2}$ as the bunch travels along the accelerator.

\section{MISALIGNED FOCUSSING ELEMENTS}

If, instead of the accelerating structures, the focusing elements are displaced, the equation of motion for the beam becomes

$$
\frac{\partial^{2}}{\partial \sigma^{2}} x+\kappa^{2}[x-d(\sigma)]=\varepsilon \int_{-\infty}^{\zeta} d \zeta^{\prime} w\left(\zeta-\zeta^{\prime}\right) F\left(\zeta^{\prime}\right) x\left(\sigma, \zeta^{\prime}\right),
$$

where $d(\sigma)$ is now the displacement of the focussing elements with respect to the beamline.

Under the same assumptions as above, and applying the same formalism, the displacement of the beam is given by

$$
x(\sigma, \zeta)=\kappa^{2} \sum_{n=0}^{\infty}(-1)^{n} \varepsilon^{n} H_{n}(\zeta) \int_{0}^{\sigma} d u i_{n+1}(u) d(\sigma-u) .
$$

This expression looks similar to the one for displaced accelerating structures, but with some important differences: the sum includes the $n=0$ term and the indices for the functions $H_{n}(\zeta)$ and $i_{n+1}(\sigma)$ are different.

The mean square displacement can be calculated in a similar fashion and is given by

$$
\left\langle x^{2}(\sigma, \zeta)\right\rangle=\kappa^{4} \sum_{m=0}^{\infty} \sum_{n=0}^{\infty}(-\varepsilon)^{m+n} x_{m+1, n+1}^{2}(\sigma) H_{m}(\zeta) H_{n}(\zeta) .
$$

With the same assumptions as for misaligned structures, and for a single very short bunch, this reduces to

$$
\left\langle x^{2}(\sigma, \zeta)\right\rangle=\kappa^{4} \sum_{n=0}^{\infty} \varepsilon^{2 n} \frac{2 d_{0}^{2} \sigma_{0}}{[n !]^{2}(2 n+1)(2 \kappa)^{2 n+2}} \sigma^{2 n+1} \frac{\zeta^{4 n}}{[2 n !]^{2}} .
$$

Keeping only the first two terms:

$$
\left\langle x^{2}(\sigma, \zeta)\right\rangle=\frac{d_{0}^{2} \sigma_{0} \kappa^{2} \sigma}{2}\left[1+\frac{\varepsilon^{2} \sigma^{2} \zeta^{4}}{48 \kappa^{2}}\right] .
$$

In this case, the front of the bunch is displaced, even in the absence of coupling to the dipole mode, and its displacement varies as $\kappa \sigma^{1 / 2}$. The bunch also develops a tail that varies as $\zeta^{4}$ and whose magnitude increases as $\kappa^{-1} \varepsilon^{2} \sigma^{5 / 2}$. It can be noted that, whereas strong focusing reduces the effect of coupling between the beam and the dipole mode and inhibits the formation of a tail, it also increases the rms displacement of the bunch resulting from a misalignment of the focusing elements.

\section{REFERENCES}

[1] C. L. Bohn and J. R. Delayen, Phys. Rev. A 45, 5964 (1992).

[2] C. L. Bohn, private communication. [3] A. W. Chao, B. Richter, and C-Y Yao, NIM 178, 1 (1980). 